\title{
Survival after hospital discharge for ST-segment elevation and non-ST-segment elevation acute myocardial infarction: a population-based study
}

This article was published in the following Dove Press journal:

Clinical Epidemiology

19 July 2013

Number of times this article has been viewed

\author{
Chad E Darling' \\ Kimberly A Fisher ${ }^{2}$ \\ David D McManus 3 ,4 \\ Andrew $\mathrm{H} \mathrm{Coles}{ }^{5}$ \\ Frederick A Spencer ${ }^{5,6}$ \\ Joel M Gore ${ }^{3,4}$ \\ Robert J Goldberg ${ }^{3}$ \\ 'Department of Emergency Medicine, \\ ${ }^{2}$ Division of Pulmonary Critical \\ Care, ${ }^{3}$ Department of Quantitative \\ Health Sciences, ${ }^{4}$ Department \\ of Medicine, ${ }^{5}$ Program for Gene \\ Function and Expression, University \\ of Massachusetts Medical School, \\ Worcester, MA, USA; ${ }^{6}$ Department \\ of Medicine, McMaster University, \\ Hamilton, Ontario, Canada
}

Background: Limited recent data are available describing differences in long-term survival, and factors affecting prognosis, after ST-segment elevation myocardial infarction (STEMI) and non-ST-segment elevation myocardial infarction (NSTEMI), especially from the more generalizable perspective of a population-based investigation. The objectives of this study were to examine differences in post-discharge prognosis after hospitalization for STEMI and NSTEMI, with a particular focus on factors associated with reduced long-term survival.

Methods: We reviewed the medical records of residents of the Worcester, MA, USA metropolitan area hospitalized at eleven central Massachusetts medical centers for acute myocardial infarction (AMI) during 2001, 2003, 2005, and 2007.

Results: A total of 3762 persons were hospitalized with confirmed AMI; of these, 2539 patients (67.5\%) were diagnosed with NSTEMI. The average age of study patients was 70.3 years and $42.9 \%$ were women. Patients with NSTEMI experienced higher post-discharge death rates with 3 -month, 1-year, and 2-year death rates of $12.6 \%, 23.5 \%$, and $33.2 \%$, respectively, compared to $6.1 \%, 11.5 \%$, and $16.4 \%$ for patients with STEMI. After multivariable adjustment, patients with NSTEMI were significantly more likely to have died after hospital discharge (adjusted hazards ratio 1.28 ; 95\% confidence interval 1.14-1.44). Several demographic (eg, older age) and clinical (eg, history of stroke) factors were associated with reduced long-term survival in patients with NSTEMI and STEMI.

Conclusions: The results of this study in residents of central Massachusetts suggest that patients with NSTEMI are at higher risk for dying after hospital discharge, and several subgroups are at particularly increased risk.

Keywords: temporal trends, community-based study, STEMI/NSTEMI

\section{Introduction}

Approximately 1 million new and recurrent acute myocardial infarctions (AMI) occur annually in the United States. ${ }^{1}$ Whereas ST-segment elevation myocardial infarction (STEMI) has long been accepted terminology in the literature and clinical setting, ${ }^{2}$ the term non-STEMI (NSTEMI) became more commonly used in the mid-1990s. ${ }^{3,4}$ Classifying patients with AMI into STEMI and NSTEMI has clinical utility since each group is comprised of patients with unique clinical characteristics, different treatment approaches, and in-hospital and short-term survival. ${ }^{5-8}$

Data from randomized trials have shown that hospitalized patients with NSTEMI have a lower risk of dying during the first few weeks after an AMI, but are at higher risk for adverse cardiovascular outcomes over the long-term than patients with a STEMI. ${ }^{9,10}$ However, data derived from clinical trials may lack generalizability since
Correspondence: Robert J Goldberg Division of Epidemiology of Chronic Diseases and Vulnerable Populations, Department of Quantitative Health Sciences, University of Massachusetts Medical School, 55 Lake Avenue North Worcester, MA, USA 01655

$\mathrm{Tel}+\mathrm{I} 508856399$ I

$\mathrm{Fax}+\mathrm{I} 5088564596$

Email robert.goldberg@umassmed.edu 
these study populations generally include younger and healthier individuals than are commonly seen in communitybased investigations. Some investigations have reported no differences in 1-year mortality rates between STEMI and NSTEMI patients, ${ }^{6}$ whereas others have observed higher death rates for patients with STEMI or for those with NSTEMI. ${ }^{7,8}$ These divergent findings may be explained in part by the different sociodemographic and clinical characteristics of AMI patients being studied as well as to sampling and methodologic approaches utilized to identify the population of interest.

We have previously described the in-hospital and 1-year death rates in patients with NSTEMI and STEMI, ${ }^{11}$ but more data are needed to delineate the longer-term prognosis of patients with these two major types of AMI, particularly from a more generalizable population-based perspective. Moreover, few recent studies have examined what factors may be associated with post-discharge mortality in these patients and whether these prognostic factors differ between patients with STEMI or NSTEMI. ${ }^{6-8}$

The objectives of this study were to describe relatively contemporary post-hospital discharge all-cause death rates, and factors associated with an increased risk of dying, in patients who were discharged after an AMI from all central Massachusetts hospitals in the 2000s, further classified according to AMI type. Data from the Worcester Heart Attack Study (WHAS), an ongoing, population-based investigation describing the clinical epidemiology of AMI in residents of central Massachusetts, were used in the present investigation. ${ }^{11-13}$

\section{Methods}

The WHAS has been collecting data describing the clinical epidemiology of AMI in residents of the Worcester, MA, USA metropolitan area hospitalized at all eleven central Massachusetts medical centers on an approximate alternating yearly basis. ${ }^{11-13}$ For the present study, we included data from only the most recent hospitalized patient cohorts, namely 2001, 2003, 2005, and 2007, in order to describe overall differences, and recent trends, in the epidemiology of STEMI and NSTEMI in this large central New England population. Of the 4208 patients hospitalized during the period under study, 435 died during hospitalization and eleven were lacking information on post-discharge survival. These 446 cases were excluded from the present study. This study was approved by the Committee for the Protection of Human Subjects at the University of Massachusetts Medical School.

\section{Study population}

The methodology of the WHAS has been described elsewhere in detail. ${ }^{11-13}$ In brief, the medical records of patients at each central Massachusetts medical center who were hospitalized for possible AMI were reviewed by trained nurses and physicians and were validated according to predetermined criteria.

Patients with STEMI and NSTEMI were classified according to standardized diagnostic criteria that we have used in this study on an ongoing basis. ${ }^{11}$ A diagnosis of STEMI was made when new ST-segment elevation was present at the $\mathrm{J}$ point in two or more contiguous leads. A diagnosis of NSTEMI was accepted when, in the absence of ST-segment elevation, ischemic ST-segment or T wave changes were present for at least 24 hours with positive cardiac enzymes and/or a typical clinical presentation. From 2003 on, in the absence of electrocardiogram (ECG) abnormalities, a diagnosis of NSTEMI was accepted when elevations in various cardiac biomarker assays, including troponin, were accompanied by typical clinical presentation.

Trained nurse and physician study personnel reviewed all baseline and serial ECGs and quality control activities were routinely conducted with respect to ECG interpretation and abstraction of data from hospital medical records. Computer systems at all greater Worcester hospitals also over-read all ECGs and were independently reviewed by trained study personnel. We excluded cases of AMI that developed in the setting of trauma, as a result of a noncardiac condition, in the perioperative period, as well as cases of AMI which occurred in patients who did not reside in the Worcester metropolitan area.

\section{Data collection}

Sociodemographic, medical history, laboratory, and clinical data were abstracted from the hospital medical records of eligible patients. ${ }^{11-13}$ Patients were classified as having an initial AMI based on the review of information contained in hospital medical records and the absence of a history of prior coronary disease. ${ }^{12,13}$ Information on the development of important inpatient clinical complications was also collected based on the review of hospital medical records. These complications included atrial fibrillation, heart failure, cardiogenic shock, and stroke; uniform working definitions of each of these complications were defined in a standardized manner. ${ }^{14-17}$

Post-discharge mortality data were collected through the review of state and Social Security death certificates as well 
as by reviewing medical records for subsequent readmissions. Our primary study end point was all-cause mortality.

\section{Data analysis}

We examined differences in the characteristics of patients discharged from all greater Worcester hospitals with STEMI versus NSTEMI by utilizing $t$-tests for continuous variables and Chi-square tests for categorical variables. We examined post-discharge survival through 2009, accounting for the varying length of time patients were followed after hospital discharge. A Cox proportional hazards regression approach was utilized to examine the independent association between selected demographic and clinical factors with post-discharge mortality over the period of follow-up. Variables were included in this regression model based on a $P$-value of $\leq 0.15$ as well as on previously published associations with these factors on long-term prognosis after AMI. Variables associated with high rates of missing data, which occurred for ejection fraction, serum lipids, and body mass index, were not included in our regression models. Post-discharge sociodemographic, clinical, or medical care related variables were not included in our regression models since we did not collect further information about these prognostic factors. Hazard ratios (HRs) and accompanying 95\% confidence intervals (CIs) around various point estimates, including overall differences in long-term survival between patients with STEMI and NSTEMI, were calculated in a standardized manner. Using the graphical and numerical methods for model assessment we found that the assumption of proportional hazards was violated by two clinically relevant factors. ${ }^{18}$ Inasmuch, we conducted a sensitivity analysis using an accelerated failure time model to confirm the validity of the Cox model in light of the presence of nonproportional hazards among these key study covariates - prior medical history of heart failure and systolic blood pressure findings at the time of hospital admission. We added the $P$-values from this model to Table 2. This analysis confirmed that the violation of the proportional hazards assumption did not affect the reliability of our results. A logistic regression analysis was also carried out for purposes of examining differences in post-discharge prognosis at key long-term points (eg, 3 months, 1 year, 2 years) between patients with STEMI versus NSTEMI, with odds ratios (ORs) and accompanying 95\% CIs calculated.

\section{Results}

\section{Characteristics of study population}

A total of 3762 individuals with confirmed AMI were discharged from all metropolitan Worcester hospitals between
2001 and 2007. The average age of study patients was 70.3 years and $42.9 \%$ were women. Of these, 2539 patients (67.5\%) were diagnosed with NSTEMI.

Patients with NSTEMI were significantly older, included a greater proportion of women, and had more comorbid cardiovascular conditions, but were less likely to have presented with an initial AMI compared to patients with STEMI (Table 1). The hospital course of patients with NSTEMI was more likely to have been complicated by the development of atrial fibrillation and heart failure (Table 1). Patients with NSTEMI had higher systolic blood pressure and serum glucose levels, and lower estimated glomerular filtration rate (GFR) findings, than patients with STEMI.

Expectedly, patients with STEMI were more likely to have undergone a percutaneous coronary intervention (PCI), whereas coronary artery bypass graft (CABG) surgery was performed in a similar percentage of both patient groups. Patients with NSTEMI were more likely to have been discharged from the hospital on digoxin and calcium channel blockers, while patients with STEMI were more likely to have been discharged on aspirin, beta-blockers, lipid-lowering agents, and angiotensin-converting-enzyme (ACE)-inhibitors/angiotensin receptor blockers.

\section{Post-hospital discharge prognosis}

Patients discharged from greater Worcester hospitals after NSTEMI during the years under study experienced higher post-discharge all-cause death rates with 3-month, 1-year, and 2-year death rates of $12.6 \%, 23.5 \%$, and $33.2 \%$, respectively, compared with $6.1 \%, 11.5 \%$, and $16.4 \%$ for patients with STEMI (Figure 1). For our initial two study cohorts (2001 and 2003) in which longer-term follow-up was available, the 5 -year death rates were $30.2 \%$ and $52.4 \%$, respectively, for patients with STEMI and NSTEMI (Figure 1).

After adjusting for baseline differences in age, sex, length of stay, comorbidities, hospital clinical complications, and physiologic variables, patients with STEMI were significantly more likely to have survived at 3 months (OR 1.38; 95\% CI 1.01-1.87), 1 year (OR 1.38; 95\% CI 1.09-1.74), and 2 years (OR 1.53; 95\% CI 1.23-1.89) (all $P$-values $<0.05$ ) after hospital discharge compared to patients with NSTEMI. Overall, patients with NSTEMI were significantly more likely to have died during the years under study than patients with STEMI (adjusted HR $=1.28 ; 95 \%$ CI 1.14-1.44) $(P$-value $<0.05)$.

Since early coronary revascularization is recommended for patients with STEMI, we carried out an additional subgroup analysis in which we examined differences in post discharge death rates in patients with STEMI and NSTEMI 
Table I Characteristics of patients with AMI according to type of AMI

\begin{tabular}{|c|c|c|c|}
\hline & $\begin{array}{l}\text { STEMI } \\
(\mathrm{n}=\mid \mathbf{2 2 3})\end{array}$ & $\begin{array}{l}\text { NSTEMI } \\
(\mathrm{n}=2539)\end{array}$ & $P$-value \\
\hline \multicolumn{4}{|l|}{ Demographics } \\
\hline Age (mean, years) (SD) & $65.5(14.3)$ & $72.6(13.6)$ & $<0.001$ \\
\hline Body mass index (mean) & $28.1(5.8)$ & $27.9(6.2)$ & 0.40 \\
\hline \multirow[t]{2}{*}{ Length of hospital stay (mean, days) (SD) } & $5.5(5.1)$ & $5.9(6.2)$ & 0.08 \\
\hline & n (\%) & n (\%) & \\
\hline Male & $778(63.6)$ & $1370(54.0)$ & $<0.001$ \\
\hline White race & $1093(89.4)$ & $2259(89.0)$ & 0.71 \\
\hline \multicolumn{4}{|l|}{ Medical history } \\
\hline Atrial fibrillation & $86(7.0)$ & $422(16.6)$ & $<0.001$ \\
\hline Diabetes & $305(25.0)$ & $952(37.5)$ & $<0.001$ \\
\hline Heart failure & $140(11.5)$ & $746(29.4)$ & $<0.001$ \\
\hline Hypertension & $765(62.6)$ & $1967(77.5)$ & $<0.001$ \\
\hline Renal disease & 145 (1 I.9) & $54 \mid(2 \mid .3)$ & $<0.001$ \\
\hline Stroke & $85(7.0)$ & $338(13.3)$ & $<0.001$ \\
\hline Initial MI & $913(74.7)$ & $1539(60.6)$ & $<0.001$ \\
\hline Hospital clinical complications & n (\%) & n (\%) & \\
\hline Atrial fibrillation & $209(17.1)$ & $543(21.4)$ & $<0.01$ \\
\hline Heart failure & $350(28.6)$ & $1050(41.4)$ & $<0.001$ \\
\hline Cardiogenic shock & $81(6.6)$ & $48(1.9)$ & $<0.001$ \\
\hline Stroke & II (0.9) & $32(1.3)$ & 0.33 \\
\hline Physiologic variables at hospital admission & mean (SD) & & \\
\hline Systolic blood pressure $(\mathrm{mmHg})$ & $139.0(31.3)$ & 145.8 (32.9) & $<0.001$ \\
\hline Diastolic blood pressure $(\mathrm{mmHg})$ & $78.9(20.6)$ & $77.1(20.5)$ & 0.02 \\
\hline Glucose $(\mathrm{mg} / \mathrm{dL})$ & $170.0(85.5)$ & $177.8(108.7)$ & 0.03 \\
\hline Total cholesterol (mg/dL) & $176.4(47.1)$ & $172.8(52.9)$ & 0.10 \\
\hline Glomerular filtration rate $\left(\mathrm{mL} / \mathrm{min} / \mathrm{I} .73 \mathrm{~m}^{2}\right)$ & $67.0(26.5)$ & $56.9(24.2)$ & $<0.001$ \\
\hline Ejection fraction (\%) & $46.2(12.9)$ & $47.2(14.1)$ & 0.11 \\
\hline Medications at time of discharge & n (\%) & n (\%) & \\
\hline Aspirin & 1085 (88.7) & $2111(83.1)$ & $<0.001$ \\
\hline Beta-blocker & $1066(87.2)$ & $2108(83.0)$ & $<0.001$ \\
\hline Anticoagulants & $177(14.5)$ & $426(16.8)$ & 0.07 \\
\hline ACE-inhibitor or angiotensin receptor blocker & $836(68.4)$ & $1469(57.9)$ & $<0.001$ \\
\hline Calcium channel blocker & $87(7.1)$ & $450(17.7)$ & $<0.001$ \\
\hline Digoxin & $112(9.2)$ & $407(16.0)$ & $<0.001$ \\
\hline Lipid lowering agents & $908(74.2)$ & $1696(66.8)$ & $<0.001$ \\
\hline \multicolumn{4}{|l|}{ Procedures during hospitalization } \\
\hline Percutaneous coronary intervention & $807(66.0)$ & $841(33.1)$ & $<0.001$ \\
\hline Coronary bypass graft surgery & $86(7.0)$ & $168(6.6)$ & 0.63 \\
\hline
\end{tabular}

Abbreviations: ACE, angiotensin-converting-enzyme; AMI, acute myocardial infarction; MI, myocardial infarction; NSTEMI, non-ST-segment elevation myocardial infarction; SD, standard deviation; STEMI, ST-segment elevation myocardial infarction.

who underwent either PCI or CABG. The results of this crude unadjusted analysis revealed death rates of $3.1 \%$ versus $3.2 \%$ at 3 months, $6.0 \%$ versus $8.4 \%$ at 1 year, and $7.9 \%$ versus $12.8 \%$ at 2 years in patients with STEMI versus NSTEMI, respectively, in patients who underwent coronary revascularization.

\section{Factors associated with post-hospital discharge prognosis}

After multivariate adjustment for the sociodemographic characteristics, comorbidities, hospital clinical complications, and admission physiologic variables included in Table 1, factors significantly associated with an adverse long-term prognosis following hospital discharge for patients with STEMI included older age, longer hospital stay, previously diagnosed heart failure, diabetes or stroke, development of atrial fibrillation during hospitalization, elevated systolic blood pressure and blood glucose findings at the time of hospital admission, and lower serum estimated GFR findings (Table 2). Among patients with NSTEMI, older individuals, men, those with a longer hospital stay, patients with a history of either stroke, heart failure, or diabetes, and those who developed stroke or 


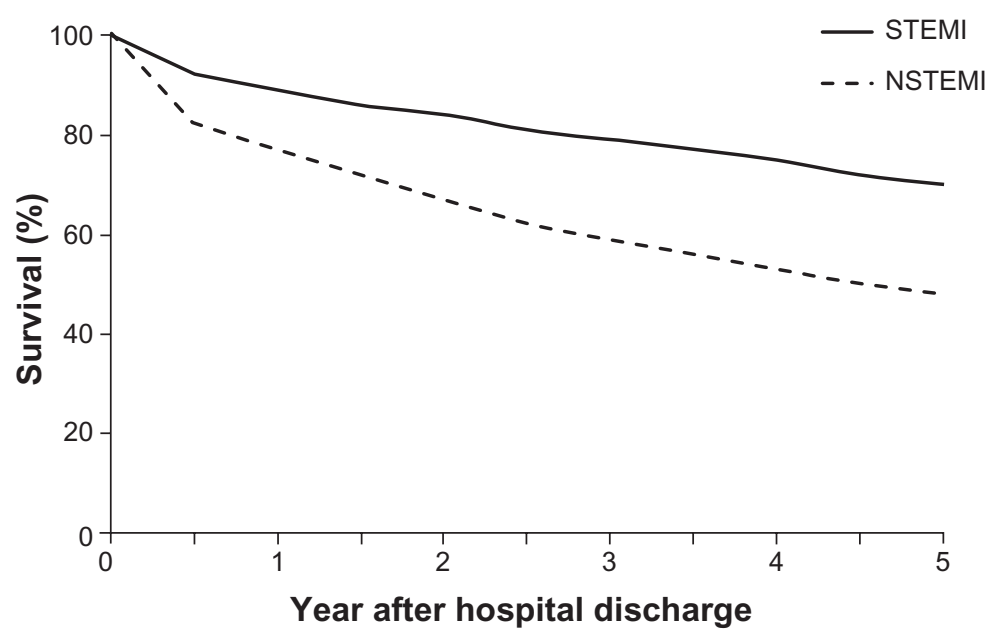

Figure I Post-discharge survival of patients according to type of AMI for patients enrolled in the Worcester Heart Attack Study in 200I, 2003,2005 , and 2007. Note: Worcester Heart Attack Study data from. ${ }^{11,12,14}$

Abbreviations: AMI, acute myocardial infarction; NSTEMI, non-ST-segment elevation myocardial infarction; STEMI, ST-segment elevation myocardial infarction.

heart failure during hospitalization were at greater risk for dying than respective comparison groups. Elevated systolic blood pressure and elevated blood glucose levels at the time of hospital presentation as well as lower serum estimated GFR findings were associated with decreased long-term mortality following NSTEMI (Table 2).

\section{Discussion}

The results of this community-based study demonstrate that the post-discharge death rates for patients hospitalized with NSTEMI were higher than for patients discharged after STEMI. After multivariate adjustment, increasing age was an important predictor of death for both patient groups after

Table 2 Factors associated with post-discharge mortality according to type of AMI

\begin{tabular}{|c|c|c|c|c|c|c|}
\hline \multirow[t]{2}{*}{ Factor } & \multicolumn{3}{|l|}{ STEMI } & \multicolumn{3}{|c|}{ NSTEMI } \\
\hline & $\begin{array}{l}\text { Hazard } \\
\text { ratio }\end{array}$ & $95 \% \mathrm{Cl}$ & $P$-value* & $\begin{array}{l}\text { Hazard } \\
\text { ratio }\end{array}$ & $95 \% \mathrm{Cl}$ & $P$-value* \\
\hline Age (per 10 year increase) & 1.66 & $1.51-1.82$ & $<0.001$ & 1.76 & $1.66-1.87$ & $<0.001$ \\
\hline Male & 1.14 & $0.92-1.42$ & 0.35 & 1.24 & $1.10-1.38$ & $<0.01$ \\
\hline Length of stay (days) & 1.03 & $1.01-1.05$ & $<0.001$ & 1.00 & $1.00-1.02$ & $<0.01$ \\
\hline \multicolumn{7}{|l|}{ Comorbidities } \\
\hline Hypertension & $\mathrm{I} .0 \mathrm{I}$ & $0.80-1.28$ & 0.60 & 0.93 & $0.80-1.07$ & $<0.01$ \\
\hline Atrial fibrillation & 1.25 & $0.91-1.71$ & 0.23 & 1.13 & $0.98-1.30$ & 0.57 \\
\hline Stroke & 1.42 & $1.04-1.94$ & $<0.05$ & 1.45 & $1.26-1.67$ & $<0.01$ \\
\hline Congestive heart failure & 1.82 & $1.38-2.39$ & $<0.01$ & 1.72 & $1.51-1.95$ & $<0.001$ \\
\hline Diabetes & 1.21 & $0.95-1.54$ & $<0.05$ & 1.26 & I.II-I.43 & $<0.05$ \\
\hline Renal disease & 1.22 & $0.92-|.6|$ & 0.67 & 1.27 & $\mathrm{I} . \mathrm{II}-\mathrm{I} .46$ & 0.33 \\
\hline Myocardial infarction & 0.98 & $0.79-1.23$ & 0.63 & 0.91 & $0.8 \mathrm{I}-1.0 \mathrm{I}$ & 0.69 \\
\hline \multicolumn{7}{|l|}{ Hospital complications } \\
\hline Atrial fibrillation & $\mathrm{I} .34$ & I.05-I.7I & $<0.05$ & 1.01 & $0.89-1.16$ & 0.10 \\
\hline Stroke & 1.27 & $0.64-2.54$ & 0.49 & 1.38 & $0.94-2.03$ & $<0.01$ \\
\hline Cardiogenic shock & 0.83 & $0.55-1.25$ & 0.34 & 1.35 & $0.95-1.92$ & 0.71 \\
\hline Heart failure & 1.06 & $0.84-1.34$ & 0.86 & 1.45 & $1.28-1.64$ & $<0.001$ \\
\hline \multicolumn{7}{|l|}{ Admission physiologic variables } \\
\hline Systolic blood pressure (per 10 mmHg increase) & 0.99 & $0.96-1.02$ & $<0.05$ & 0.97 & $0.96-0.99$ & $<0.05$ \\
\hline \multicolumn{7}{|l|}{ Estimated glomerular filtration rate $\left(\mathrm{mL} / \mathrm{min} / \mathrm{I} .73 \mathrm{~m}^{2}\right)$} \\
\hline$<30$ & 2.68 & $1.88-3.82$ & $<0.001$ & 2.04 & $1.69-2.46$ & $<0.001$ \\
\hline $30-59$ & 1.40 & I.II-I.77 & 0.34 & 1.23 & $|.07-1.4|$ & $<0.01$ \\
\hline Elevated blood glucose levels (per 10 mg/dL increase) & 1.02 & $1.01-1.03$ & $<0.01$ & 1.00 & $1.00-1.01$ & $<0.05$ \\
\hline
\end{tabular}

Note: *P-value from accelerated failure time model.

Abbreviations: AMI, acute myocardial infarction; Cl, confidence interval; NSTEMI, non-ST-segment elevation myocardial infarction; STEMI, ST-segment elevation myocardial infarction. 
hospital discharge, whereas several factors were differentially associated with mortality depending on AMI type.

Similar to the results of other studies, ${ }^{6,7,19}$ a previous publication from the WHAS showed that patients with NSTEMI were at higher risk for dying during the first year after hospital discharge. ${ }^{11}$ In the multicenter registry of more than 2100 patients hospitalized with an AMI at 56 centers throughout France, ${ }^{6}$ patients with NSTEMI experienced higher post-discharge death rates as well as rates of rehospitalization than patients with STEMI. Among a large cohort of patients undergoing cardiac catheterization between 1999 and 2007 at Duke University Medical Center, patients who developed NSTEMI experienced a higher risk of dying over the long-term (median follow-up $=4$ years) than patients with STEMI. ${ }^{7}$ Similarly, when we extended our period of follow-up even further to 5 years after hospital discharge, patients with NSTEMI continued to fare appreciably worse over the long-term.

It has been previously reported that patients with STEMI are treated more aggressively with various inpatient therapies that are not based on disease severity. ${ }^{6}$ We similarly observed differences in the receipt of inpatient procedures and discharge medications according to AMI type. While these treatment practices may be accounted for by differential indications and current practice guidelines, it is possible that differences in treatment may have partially accounted for the improved long-term survival we observed in patients with STEMI. We did not, however, control for the receipt of either cardiac medications or coronary revascularization procedures in our regression models due to the significant potential for confounding by treatment indication in the context of this nonrandomized observational study. When we did, however, carry out a subgroup analysis in patients who underwent either a PCI or CABG surgery during hospitalization, patients with NSTEMI continued to experience higher all-cause death rates over our follow-up period than patients with STEMI.

The 1-year all-cause mortality rates for patients discharged from greater Worcester hospitals after STEMI (12\%) and NSTEMI (24\%) were slightly higher for STEMI, but considerably higher for NSTEMI, than what has been reported in prior investigations. A single-center observational study of patients with myocardial infarction undergoing PCI reported 1-year death rates of $9.5 \%$ and $14.3 \%$ for STEMI and NSTEMI, respectively. ${ }^{7}$ A study of 1486 patients with AMI enrolled in the multicenter Dynamic Registry observed 1-year mortality rates of $7.3 \%$ for STEMI and $5.5 \%$ for NSTEMI, ${ }^{20}$ while the 1-year death rates were $9.0 \%$ in STEMI patients and
$11.6 \%$ in NSTEMI patients in the OPERA registry. ${ }^{6}$ In the GUSTO-IIb trial, 1-year death rates were $9.6 \%$ for patients with STEMI and $11.1 \%$ for those with NSTEMI. ${ }^{19}$

The discrepancies between these results and our findings are likely attributable to the advanced age and comorbid disease burden of patients in our community-based study as compared with those enrolled in other studies with different methodologies and more restrictive inclusion criteria, ${ }^{19}$ or only patients studied at specialized medical centers. ${ }^{7}$ Our findings may be more generalizable to the overall population of patients experiencing an AMI, and are important as they help to resolve the discrepancies in the results between other studies performed over a similar time period in more select patient populations. ${ }^{7,20}$ The present results lend further support to the notion that patients discharged from the hospital after NSTEMI constitute a high risk group in whom close surveillance and the use of evidence-based cardiac medications, where appropriate, is warranted.

\section{Factors associated with increased mortality}

In a multicenter study of patients hospitalized with AMI at 56 centers in France in the early 2000s, while advanced age, high heart rate on admission, and low levels of blood pressure at the time of hospital admission were independent, and similar, correlates of 1-year death rates in patients with STEMI or NSTEMI, differences in the predictability of several factors, or their strength of association, were observed in patients according to type of AMI. ${ }^{6}$

Results of prior studies examining the association between sex and mortality after AMI have been conflicting, especially after controlling for age. ${ }^{21,22}$ In the present study, men were more likely to have died after hospital discharge for NSTEMI and STEMI.

Another important prognostic factor was the presence of renal dysfunction, which was associated with long-term mortality in both patient groups. Similarly, prior studies have demonstrated higher in-hospital, 30-day, and 3-year mortality rates in patients with AMI and renal impairment as compared to those with intact renal function. ${ }^{23,24}$ Renal impairment may increase mortality through multiple mechanisms, including a decreased likelihood of receiving appropriate therapies due to concern over an increased risk of bleeding or worsening renal function, drug toxicity, and endothelial dysfunction. ${ }^{23,24}$

The long-term mortality for patients with NSTEMI, in particular, has been related to the extent of their comorbid disease burden. ${ }^{25}$ In the present study, patients with NSTEMI were more likely to have several comorbid diseases 
previously diagnosed, but patients with NSTEMI and STEMI were both found to have decreased survival if they had a history of heart failure. This is not surprising given the dismal long-term prognosis that is associated with this clinical syndrome. ${ }^{26}$ Similarly, we found that a history of prior stroke was associated with decreased survival in both patient groups after hospital discharge. This finding is consistent with prior reports where AMI patients with prior stroke were treated less aggressively, and had higher rates of complications and inpatient and early post-discharge mortality, than patients without previously diagnosed stroke. ${ }^{27}$

Interestingly, we found that patients with NSTEMI who presented with an elevated systolic blood pressure had reduced mortality. A registry study in Sweden also found that, among patients admitted to the intensive care unit with AMI, an elevated blood pressure was associated with reduced 1-year mortality. ${ }^{28}$ Why a higher systolic blood pressure might be associated with improved outcomes for patients with AMI is unknown, but may reflect preserved myocardial function or absence of systemic hypoperfusion.

Despite similarities in some prognostic factors, there were also differences in the prognostic impact of several physiologic variables and in-hospital clinical complications between patients with STEMI and NSTEMI, with a number of these factors having a particularly adverse impact in those with NSTEMI. These findings suggest the need for greater surveillance, and intervention where appropriate, for high risk patients with NSTEMI.

\section{Study strengths and limitations}

The strengths of this study include its community-based perspective, its relatively contemporary population of patients hospitalized with AMI, and its large sample size. Our study has several limitations, however, that should be considered when interpreting the present results. The greater Worcester population is predominantly Caucasian and our findings may lack generalizability to other ethnic groups or communities of varying size. We were not able to comment on the influence that hospital and post-discharge treatment practices may have had on long-term prognosis since this was a nonrandomized observational study and we did not collect information about the receipt of different medication treatment regimens or coronary revascularization approaches after a patient's discharge from all central Massachusetts hospitals. We were unable to further classify patients with AMI into a recently recommended schema ${ }^{29}$ and to satisfactorily assess, and by extension, analytically control for, other potentially important factors that may have influenced prognosis after AMI, such as infarct size, serum lipid or blood pressure levels, or presence of adverse lifestyle practices, such as cigarette smoking.

\section{Conclusion}

In this relatively contemporary cohort, patients with STEMI experienced a better post-discharge prognosis than those with NSTEMI. The factors associated with increased mortality for each of these patient groups were somewhat distinct, emphasizing the uniqueness of each disease category. Additional population-based surveillance efforts are needed to monitor recent trends in the natural history, functional status, and quality of life in patients further classified according to type of AMI. Studies leading to better understanding of the optimal inpatient and outpatient treatments based on AMI type and comorbid disease burden may maximize long-term outcomes for these patients.

\section{Acknowledgments}

This research was made possible by the cooperation of the cardiology and medical records departments of participating greater Worcester hospitals. Grant support was provided by the National Institute of Health (NIH) (RO1HL35434). Partial salary support for Drs Goldberg and McManus was provided for by NIH grant 1U01HL105268-01. Dr Darling was supported by NIH grant K23HL10199.

\section{Disclosure}

The authors report no conflicts of interest in this work.

\section{References}

1. Lloyd-Jones D, Adams RJ, Brown TM, et al; American Heart Association Statistics Committee and Stroke Statistics Subcommittee. Heart disease and stroke statistics - 2010 update: a report from the American Heart Association. Circulation. 2010;121(7):e46-e215.

2. Ross J Jr. Electorcardiographic ST-segment analysis in the characterization of myocardial ischemia and infarction. Circulation. 1976;53(3 Suppl): I73-I81.

3. Braunwald E, Antman EM, Beasley JW, et al. ACC/AHA guidelines for the management of patients with unstable angina and non-STsegment elevation myocardial infarction: executive summary and recommendations. A report of the American College of Cardiology/ American Heart Association task force on practice guidelines (committee on the management of patients with unstable angina). Circulation. 2000;102(10):1193-1209.

4. Braunwald E, Jones RH, Mark DB, et al. Diagnosing and managing unstable angina. Agency for Health Care Policy and Research. Circulation. 1994;90(1):613-622.

5. Anderson JL, Adams CD, Antman EM, et al; 2011 WRITING GROUP MEMBERS; ACCF/AHA TASK FORCE MEMBERS. 2011 ACCF/AHA Focused Update Incorporated Into the ACC/AHA 2007 Guidelines for the Management of Patients With Unstable Angina/Non-ST-Elevation Myocardial Infarction: a report of the American College of Cardiology Foundation/American Heart Association Task Force on Practice Guidelines. Circulation. 2011;123(18):e426-e579. 
6. Montalescot G, Dallongeville J, Van Belle E, et al; OPERA Investigators. STEMI and NSTEMI: are they so different? 1 year outcomes in acute myocardial infarction as defined by the ESC/ACC definition (the OPERA registry). Eur Heart J. 2007;28(12):1409-1417.

7. Chan MY, Sun JL, Newby LK, et al. Long-term mortality of patients undergoing cardiac catheterization for ST-elevation and non-ST-elevation myocardial infarction. Circulation. 2009;119(24):3110-3117.

8. Terkelsen CJ, Lassen JF, Nørgaard BL, et al. Mortality rates in patients with ST-elevation vs non-ST-elevation acute myocardial infarction: observations from an unselected cohort. Eur Heart J. 2005;26(1): $18-26$.

9. Cantor WJ, Goodman SG, Cannon CP, et al. Early cardiac catheterization is associated with lower mortality only among high-risk patients with ST- and non-ST-elevation acute coronary syndromes: observations from the OPUS-TIMI 16 trial. Am Heart J. 2005;149(2):275-283.

10. Cox DA, Stone GW, Grines CL, et al; CADILLAC Investigators. Comparative early and late outcomes after primary percutaneous coronary intervention in ST-segment elevation and non-ST-segment elevation acute myocardial infarction (from the CADILLAC trial). Am J Cardiol. 2006;98(3):331-337.

11. McManus DD, Gore J, Yarzebski J, Spencer F, Lessard D, Goldberg RJ. Recent trends in the incidence, treatment, and outcomes of patients with STEMI and NSTEMI. Am J Med. 2011;124(1):40-47.

12. Floyd KC, Yarzebski J, Spencer FA, et al. A 30-year perspective (1975-2005) into the changing landscape of patients hospitalized with initial acute myocardial infarction: Worcester Heart Attack Study. Circ Cardiovasc Qual Outcomes. 2009;2(2):88-95.

13. Goldberg RJ, Spencer FA, Yarzebski J, et al. A 25-year perspective into the changing landscape of patients hospitalized with acute myocardial infarction (the Worcester Heart Attack Study). Am J Cardiol. 2004;94(11):1373-1378.

14. McManus DD, Chinali M, Saczynski JS, et al. 30-year trends in heart failure in patients hospitalized with acute myocardial infarction. Am J Cardiol. 2011;107(3):353-359.

15. Saczynski JS, McManus D, Zhou Z, et al. Trends in atrial fibrillation complicating acute myocardial infarction. Am J Cardiol. 2009;104(2): 169-174.

16. Goldberg RJ, Spencer FA, Gore JM, Lessard D, Yarzebski J. Thirty year trends (1975-2005) in the magnitude of, management of, and hospital death rates associated with cardiogenic shock in patients with acute myocardial infarction: A population-based perspective. Circulation. 2009;119(9):1211-1219.

17. Saczynski JS, Spencer FA, Gore JM, et al. Twenty-year trends in the incidence of stroke complicating acute myocardial infarction: The Worcester Heart Attack Study. Arch Intern Med. 2008;168(19):2104-2110.
18. Lin D, Wei LJ, Ying Z. Checking the Cox model with cumulative sums of martingale-based residuals. Biometrika. 1993;80(3):557-572.

19. Armstrong PW, Fu Y, Chang WC, et al. Acute coronary syndromes in the GUSTO-IIb trial: prognostic insights and impact of recurrent ischemia. The GUSTO-IIb Investigators. Circulation. 1998;98(18):1860-1868.

20. Abbott JD, Ahmed HN, Vlachos HA, Selzer F, Williams DO. Comparison of outcome in patients with ST-elevation versus non-STelevation acute myocardial infarction treated with percutaneous coronary intervention (from the National Heart, Lung, and Blood Institute Dynamic Registry). Am J Cardiol. 2007;100(2):190-195.

21. Berger JS, Elliott L, Gallup D, et al. Sex differences in mortality following acute coronary syndromes. JAMA. 2009;302(8):874-882.

22. Champney KP, Frederick PD, Bueno H, et al; NRMI Investigators. The joint contribution of sex, age and type of myocardial infarction on hospital mortality following acute myocardial infarction. Heart. 2009;95(11):895-899.

23. Freeman RV, Mehta RH, Al Badr W, Cooper JV, Kline-Rogers E, Eagle KA. Influence of concurrent renal dysfunction on outcomes of patients with acute coronary syndromes and implications of the use of glycoprotein IIb/IIIa inhibitors. J Am Coll Cardiol. 2003;41(5): $718-724$.

24. Anavekar NS, McMurray JJ, Velazquez EJ, et al. Relation between renal dysfunction and cardiovascular outcomes after myocardial infarction. N Engl J Med. 2004;351(13):1285-1295.

25. Allen LA, O'Donnell CJ, Camargo CA Jr, Giugliano RP, LloydJones DM. Comparison of long-term mortality across the spectrum of acute coronary syndromes. Am Heart J. 2006;151(5):1065-1071.

26. Goldberg RJ, Darling C, Joseph B, et al. Epidemiology of decompensated heart failure in a single community in the northeastern United States. Am J Cardiol. 2009;104(3):377-382.

27. Cooper HA, Domanski MJ, Rosenberg Y, et al; Magnesium in Coronaries trial investigators. Acute ST-segment elevation myocardial infarction and prior stroke: an analysis from the Magnesium in Coronaries (MAGIC) trial. Am Heart J. 2004;148(6):1012-1019.

28. Stenestrand U, Wijkman M, Fredrikson M, Nystrom FH. Association between admission supine systolic blood pressure and 1-year mortality in patients admitted to the intensive care unit for acute chest pain. JAMA. 2010;303(12):1167-1172.

29. Thygesen K, Alpert JS, Jaffe AS, Simoons ML, Chaitman BR, White HD. Third universal definition of myocardial infarction. Eur Heart J. 2012;33:2551-2567.
Clinical Epidemiology

\section{Publish your work in this journal}

Clinical Epidemiology is an international, peer-reviewed, open access journal focusing on disease and drug epidemiology, identification of risk factors and screening procedures to develop optimal preventative initiatives and programs. Specific topics include: diagnosis, prognosis, treatment, screening, prevention, risk factor modification, systematic

Submit your manuscript here: http://www.dovepress.com/clinical-epidemiology-journal

\section{Dovepress}

reviews, risk \& safety of medical interventions, epidemiology \& biostatical methods, evaluation of guidelines, translational medicine, health policies \& economic evaluations. The manuscript management system is completely online and includes a very quick and fair peer-review system, which is all easy to use. 